\title{
FINE STRUCTURE OF RESONANT-TUNNELING PEAK IN GaAs/AlAs DOUBLE-BARRIER HETEROSTRUCTURE*
}

\author{
S.A. Vitusevich ${ }^{\dagger}$, T. Figielski, A. MąKosa, T. Wosiński
}

Institute of Physics, Polish Academy of Sciences

Al. Lotników 32/46, 02-668 Warszawa, Poland

A.E. Belyaev, R.V. Konakova

Institute of Semiconductor Physics, National Academy of Sciences of Ukraine 252028 Kiev, Ukraine

and L.N. KRavchenko

Science and Research Institute of Molecular Electronics, 103460 Moscov, Russia

For the first time we observed a fine oscillatory structure, with the period of $36 \mathrm{mV}$, of the resonant tunneling peak in the current-voltage characteristic of a double-barrier heterostructure. We attribute it to a sequential single-phonon emission of ballistic electrons which tunneled out from the quantum well through the collector barrier.

PACS numbers: 73.40.Gk, 85.30.Mn

We investigated an asymmetric double-barrier resonant-tunneling structure grown by molecular beam epitaxy. It consisted basically of two narrow $2 \mathrm{~nm}$ AlAs barriers (for such thin barriers direct $\Gamma$ electron tunneling is the dominant process [1]) separated by a $4 \mathrm{~nm}$ GaAs quantum well, and had incorporated a $100 \mathrm{~nm}$ undoped GaAs spacer layer adjacent to one of the barriers. The GaAs emitter and collector regions were doped to $n=10^{18} \mathrm{~cm}^{-3}$. Two quasi-bound states of the well are expected to appear in this structure: $E_{0}=0.18 \mathrm{eV}$ and $E_{1}=0.68 \mathrm{eV}$. We measured the current-voltage characteristic $I(V)$, and differential conductance $\mathrm{d} I / \mathrm{d} V$, of square mesa diodes of the area $[32 \mu \mathrm{m}]^{2}$ fabricated from this structure.

Surprisingly, three resonant peaks in the $I(V)$ curve were observed at low temperature when electrons were injected from the spacer side (forward bias), instead of two peaks that actually appeared when the bias polarity was reversed (Fig. 1). The above peculiarity seems to infer from the fact that under the for-

*This work was supported by the State Committee for Scientific Research (Republic of Poland), project PBZ-101-01-11.

t On leave from the Institute of Semiconductor Physics, National Academy of Sciences of Ukraine, Kiev, Ukraine. 


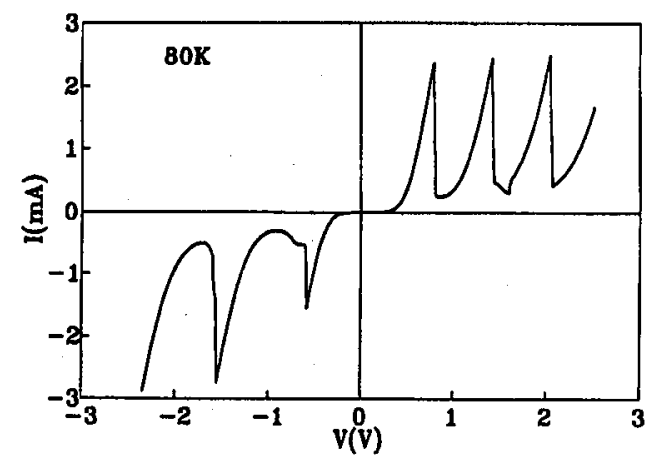

Fig. 1. Current--voltage characteristic of the resonant-tunneling diode under two bias polarities.

ward bias electrons can be injected from an $n^{+}$-type emitter into the well in two different ways: either ballistically or via a subband of a $2 \mathrm{D}$ accumulation region formed in the spacer layer [2]. Under a small bias, when the energy difference between the conduction-band edge in the emitter region and the subband edge in the accumulation layer is small, these two ways are not distinguishable in the resonant tunneling via the $E_{0}$ state. Instead, under a large bias this difference becomes significant and gives rise to a separation of the resonant peak, associated with the tunneling via the $E_{1}$ state, into two different peaks. One of them is due to electrons transported ballistically between the emitter and the first barrier and it ceases at elevated temperature when phonon scattering shortens the mean free path of electrons. The other is due to electrons thermalized in the subband of the accumulation layer, prior to the resonant tunneling.

At each peak the current increases steadily with applied voltage up to the maximum and then drops down to a non-resonant value, following the load line in series equivalent of the measuring circuit. A novel feature of the resonant peaks was discovered at low temperature while measuring the $\mathrm{d} I / \mathrm{d} V$ vs. $V$ relationship. Below $\approx 120 \mathrm{~K}$ a fine oscillatory structure appears on the increasing slope of the resonant peaks, independently of the bias polarity. Up to 12 cycles of the oscillations could be distinguished, having a period of about $36 \mathrm{mV}$ (Fig. 2). The amplitude of the oscillations increases with lowering temperature and is proportional to the current intensity at a given bias. This structure is probably associated with LO phonons in GaAs, whose energy is just $36 \mathrm{meV}$, but there is no obvious mechanism which leads to their appearing. It is important to realize that the observed oscillatory structure must essentially represent a relationship between the tunnel current and the voltage drop across the second (collector) barrier, as it is discussed below.

In the voltage range where a resonant peak appears, a change in the voltage drop across the first (emitter) barrier cannot exceed the peak width which presumedly amounts to some tens of millivolts in our case. But the observed range of the increasing slope of the peaks is much more extended while measuring versus applied bias voltage, owing to a screening effect caused by an electronic space-charge 


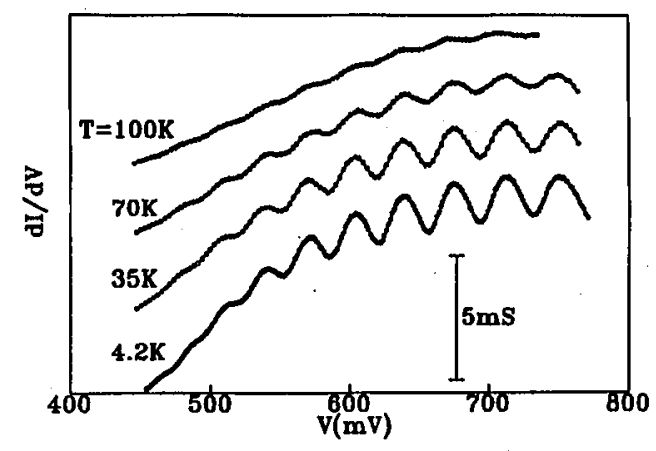

Fig. 2. Fine structure of the increasing slope of a resonant peak of the diode measured at different temperatures.

built-up in the quantum well. It can be shown that for a large space-charge the intrinsic $I(V)$ curve should display a range of applied voltages where the current is triple valued [3,4], but the latter can hardly be observed because of a competitive bistable behavior of the device in this range.

Our proposal for the explanation of the observed oscillatory structure invokes the phenomenon of sequential single-phonon emission by ballistic electrons which resonantly tunneled throughout the barriers. A phenomenon of this kind has already been observed in the case of tunneling through a single potential barrier, but only under very critical experimental conditions $[5,6]$.

We suppose that an electron which escaped from the well by the tunneling through the collector barrier loses its energy owing to a sequential emission of single LO phonons, dissipating an energy quantum $\hbar \omega \approx 36 \mathrm{meV}$ in each act of emission. Depending on the energy difference between the quasi-bound state of the well and the quasi-Fermi level in the collector region, an electron after several acts of emission becomes either fully thermalized or still keeps some excess energy that is, however, unsufficient to be lost in one more act of LO-phonon emission. Such an electron moves further ballistically until some other mechanisms of scattering lead it down to the quasi-Fermi level. It contributes to the conductance of the beyond-the-barrier region in a different way than a fully thermalized electron, thus giving rise to a slightly different potential distribution across the structure. This effect eventually results in the oscillatory fine structure of the resonant-tunneling current.

To complete this communication, we have to emphasize that the origin of the reported structure seems to be completely different from another one, also connected with LO phonons, which we have found to appear in the broad plateau region of heterostructures displaying so-called double-step feature in the $I(V)$ curve $[7,8]$.

\section{Acknowledgment}

The authors are indebted to Dr. E. Kaczmarek for helpful discussions. 


\section{References}

[1] D. Landheer, H.C. Liu, M. Buchanan, R. Stoner, Appl. Phys. Lett. 54, 1784 (1989).

[2] P.J. Price, Phys. Rev. B 45, 9042 (1992).

[3] F.W. Sheard, G.A. Toombs, Appl. Phys. Lett. 52, 1228 (1988).

[4] Y. Abe, Semicond. Sci. Technol. 7, 8498 (1992).

[5] T.W. Hickmott, P.M. Solomon, F.F. Fang, F. Stern, Phys. Rev. Lett. 52, 2053 (1984).

[6] A. Alikacem, D.K. Maude, M. Henini, L. Eaves, G. Hill, M.A. Pate, Semicond. Sci. Technol. 7, 8446 (1992).

[7] T. Figielski, A. Mąkosa, T. Wosiński, P.C. Harness, K.E. Singer, Acta Phys. Pol. A 84, 817 (1993).

[8] T. Figielski, A. Mąkosa, T. Wosiński, P.C. Harness, K.E. Singer, Solid State Commun. 91, 913 (1994). 